\title{
COORDENAÇÃO EM SISTEMAS AGROALIMENTARES: UM LEVANTAMENTO BIBLIOMETRICO ENTRE 2009 E 2015
}

\author{
COORDINATION IN AGRIFOOD SYSTEMS: \\ A BIBLIOMETRIC STUDY BETWEEN 2009 AND 2015
}

\section{Amanda Ferreira Guimarães}

Doutoranda em Administração pela Universidade Estadual de Maringá (Maringá/Brasil).

E-mail: amandafguimaraes@live.com

\section{Elisa Mirales}

Mestre em Administração pela Universidade Estadual de Maringá (Maringá/Brasil).

E-mail: mirales.elisa@gmail.com

\section{Rejane Heloise dos Santos}

Doutoranda em Administração pela Universidade Estadual de Maringá (Maringá/Brasil).

E-mail: rejaneheloise@hotmail.com

\section{Sandra Mara de Alencar Schiavi}

Pós-Doutora em Economia Agrícola pela Universidade Estadual do Kansas (Nova lorque/Estados Unidos da América).

Professora do Departamento de Administração e dos Programas de Pós-Graduação em Administração (PPA/UEM)

e em Economia (PCE/UEM) na Universidade Estadual de Maringá (Maringá/Brasil).

E-mail: smsbankuti@uem.br 


\section{RESUMO}

Aose tratarde sistemas agroalimentares da pecuária de corte, coexistem sistemas convencionais ediferenciados, envolvendo uma multiplicidade de definições. Nesse sentido, o objetivo do presente artigo foi realizar um levantamento da produção de conhecimento acerca do tema "Coordenação de Sistemas Agroalimentares" nos últimos sete anos, de modo a fornecer contribuições para a compreensão contextualizada da produção e fundamentar a agenda de pesquisas futuras sobre o tema. Realizou-se um levantamento bibliométrico seguido de análise descritiva e analítica. Os resultados apontam para a predominância de estudos com foco em sistemas convencionais, porém, apresenta oportunidades para estudos em sistemas diferenciados. A carne bovina e a pecuária de corte estão entre os objetos mais pesquisados, sendo a principal abordagem a pesquisa qualitativa por meio de estudos de casos. Sobre o tema, estudos internacionais se concentram mais em pesquisas quantitativas quando comparados ao Brasil, mostrando maior interesse nessa abordagem. As principais teorias utilizadas são a Teoria dos Custos de Transação, a Teoria dos Custos de Mensuração e a Teoria de Redes, bem como seus respectivos autores. Os principais objetivos dos estudos estão associados à compreensão dos diversos mecanismos de coordenação, envolvendo confiança, formas hỉbridas, inovação. Verificou-se que a coexistência de dois subsistemas, ao se tratar de sistemas agroalimentares, e a diversidade de caminhos para estudo do tema se constituem como indicativos para estudos que possam contribuir para o avanço das pesquisas sobre sistemas agroalimentares.

Palavras-chave: Estudo bibliométrico. Sistemas Agroalimentares. Coordenação.

\section{ABSTRACT}

When dealing with agrifood systems of beef cattle, conventional and differentiated systems coexist, involving a multiplicity of definitions. This article aims to conduct a survey of the production of knowledge on the theme "Coordination of Agrifood Systems" in the last seven years., in order to provide contributions to the contextualized understanding of production and to base the agenda for future research on theme. A bibliometric survey was performed followed by descriptive and analytical analysis. The results point to the predominance of studies focusing on conventional systems, however, presents opportunities for studies in differentiated systems. Beef and beef cattle are among the most researched objects, being the main approach to qualitative research through case studies. On the subject, international studies focus more on quantitative research when compared to Brazil, showing greater interest in this approach. The main theories used are the Transaction Cost, Measurement Cost and the Network Theory, as well as their respective authors. The main objectives are associated with the understanding of the various coordination mechanisms, involving trust, hybrid forms, innovation It was found that the coexistence of two agri-food, and the diversity of ways to study the theme, are indicative for studies that can contribute to the advancement of research on agri-food systems.

Keywords: Bibliometric study. Agrifood systems. Coordination. 


\section{INTRODUÇÃO}

O agronegócio brasileiro tem grande importância no cenário nacional e internacional. O Produto Interno Bruto (PIB) do agronegócio, em 2017, correspondeu a 21,1\% do PIB brasileiro, sendo a pecuária responsável por 24,1\% desse resultado (CEPEA, 2019). No entanto, apesar dos números favoráveis, é possivel observar diversos problemas de competitividade nesse SAG, especialmente ao se considerar falhas de coordenação na cadeia agroindustrial da carne bovina (PIGATTO et al., 1999; IPARDES, 2002; ZYLBERSZTAJN; MACHADO FILHO, 2003; BARCELLOS et al., 2004; FERREIRA; BARCELLOS, 2004; BUAINAIN; BATALHA, 2007; CALEMAN et al., 2008; SAAB et al., 2009; CALEMAN; ZYLBERSZTAJN, 2012; OLIVEIRA JÚNIOR et al., 2020).

Pesquisas apontam que grande parte desses sistemas adotam estruturas de governança do tipo spot(CALEMAN, et al., 2008; PASCOAL et al., 2011; CALEMAN; ZYLBERSZTAJN, 2012; CARRER et al., 2014), sendo caracterizados pela comercialização de produtos commodity. Nesse tipo de transação os preços são geralmente definidos pela indústria, seguindo o comportamento de oferta e demanda (PASCOAL et al., 2011; CALEMAN; ZYLBERSZTAJN, 2012). As relações entre produtores e compradores nos sistemas agroindustriais da carne bovina são majoritariamente caracterizadas pela presença de oportunismo, no qual o foco tem sido o lucro a curto prazo, visando a manutenção da lucratividade da indústria (FERREIRA; PADULA, 2002; BUAINAIN; BATALHA, 2007; SAAB et al., 2009; PASCOAL et al., 2011; OLIVEIRA et al., 2015).

Pascoal et al. (2011) defendem que uma forma de alcançar a redução dos conflitos na cadeia da carne bovina é mediante a descommoditização do produto, uma vez que diferenciações pelos esforços na produção exigem maior coesão e coordenação entre os agentes. No SAG da carne bovina, isso pode ser obtido pela formação de sistemas diferenciados, tais como aqueles associados a raças específicas ou a sistemas de produção alternativos, como o orgânico, denominados Sistemas Agroalimentares Diferenciados (SAD). Segundo Bánkuti (2014), entende-se que um SAD se constitui como um subsistema cuja organização das atividades produtivas e dos agentes é voltada para diferenciação, seja por meio de produtos diferenciados, processos alternativos ou por canais diferenciados de distribuição, associando atividades no segmento da produção rural.

No que tange à pecuária bovina de corte do Brasil, é possivel observar dois sistemas: aquele caracterizado pela produção de carne bovina do tipo commodity e aquele com foco em diferenciação. Apesar dos esforços em oferecer alternativas, perduram as falhas de coordenação na cadeia da carne bovina. Todavia, ao se considerar as particularidades de SADs, tais como aqueles voltados para a produção de carnes com algum apelo de diferenciação, o modo como produtores e compradores organizam as trocas 
na cadeia se torna algo latente para competitividade (MACEDO; MORAES, 2009; MALAFAIA et al., 2009; CALEMAN; ZYLBERSZTAJN, 2011). Sistemas caracterizados por baixa qualidade (atender às demandas de preço e quantidade) e alta qualidade (características de diferenciação) exigem mecanismos de governança diferentes (ZYLBERSZTAJN; MACHADO FILHO, 2003).

Dada a coexistência de sistemas agroalimentares diferenciados e convencionais e a necessidade de estudos sobre o tema, principalmente no que se refere àqueles relacionados à carne bovina e à pecuária de corte, o presente trabalho teve como objetivo realizar um levantamento da produção de conhecimento acerca do tema "Coordenação de Sistemas Agroalimentares" nos últimos sete anos. O diagnóstico desses dados é fundamental para que futuras pesquisas possam ser feitas com maior coerência, consistência, validade e confiabilidade. Nesse sentido, o presente estudo pretende contribuir para a compreensão contextualizada da produção científica no que diz respeito aos sistemas agroalimentares, de modo a fundamentar a agenda de pesquisas futuras sobre o tema.

Para tanto, o artigo conta com quatro seções. A partir desta introdução aqui apresentada, a segunda visa apresentar a metodologia para a obtenção do objetivo de pesquisa. A terceira é composta pela análise descritiva e analítica dos dados. E, por fim, a quarta e última seção apresenta as conclusões.

\section{METODOLOGIA}

Para que o objetivo da presente pesquisa fosse alcançado, o método de levantamento bibliométrico foi adotado. Alan Pritchard, em 1969, propôs o termo bibliometria (bibliometrics) em substituição ao termo bibliografia estatística. $O$ autor argumenta que a bibliometria é o estudo realizado mediante a aplicação de métodos estatísticos e matemáticos a diferentes meios de comunicação (PRITCHARD, 1969; SILVA; BIANCHI, 2001). Segundo Chueke e Amatucci (2015), um levantamento bibliométrico é especialmente importante, pois ele permite a sistematização dos estudos científicos realizados sobre determinado tema e também a identificação de campos de pesquisas futuras. A principal justificativa para a escolha do levantamento bibliométrico foi o tamanho da amostra, que foi maior do que 40 trabalhos (CHUEKE; AMATUCCl, 2015).

A execução do levantamento bibliométrico seguiu alguns passos planejados e programados. A primeira etapa consistiu em um levantamento teórico sobre o presente método, buscando compreender quais as características e diretrizes coerentes com o instrumento para evitarem-se as falhas. No segundo momento, a partir de uma revisão bibliográfica sobre o tema "Coordenação de Sistemas Agroalimentares", os termos a serem buscados foram definidos seguindo os critérios de coerência com o tema de pesquisa. Assim, as palavras-chave envolveram não somente o próprio tema, mas, também aquelas que são 
caracterizadas como sendo características de Sistemas Diferenciados. Os termos, que foram consultados tanto em sua forma singular quanto plural, totalizaram 25 palavras para busca, como pode ser observado no Quadro 01.

\begin{tabular}{|l|l|}
\hline \multicolumn{2}{|c}{ Quadro 01: Termos de Pesquisa } \\
\hline \multirow{2}{*}{ Sistema Diferenciado } & Não convencional \\
\cline { 2 - 2 } & Não convencionais \\
\hline \multirow{2}{*}{ Sistemas Diferenciados } & Orgânico \\
\hline \multirow{3}{*}{ Sistema de Qualidade } & Orgânicos \\
\cline { 2 - 2 } & Diferenciação \\
\hline \multirow{3}{*}{ Sistemas de Qualidade } & Diferenciações \\
\cline { 2 - 2 } & Certificação \\
\hline \multirow{3}{*}{ Sistema Alternativo } & Certificações \\
\cline { 2 - 2 } & Aliança estratégica \\
\hline \multirow{2}{*}{ Sistemas Alternativos } & Alianças estratégicas \\
\hline \multirow{3}{*}{ Sistema Agroindustrial } & Aliança Mercadológica \\
\cline { 2 - 2 } & Alianças Mercadológicas \\
\hline \multirow{3}{*}{ Sistemas Agroindustriais } & Aliança Horizontal \\
\cline { 2 - 2 } & Alianças Horizontais \\
\hline \multirow{2}{*}{ Aliness } & Aliança Vertical \\
\cline { 2 - 2 } & Alianças Verticais \\
\hline
\end{tabular}

Fonte: Elaborado pelos autores

Foram buscados trabalhos em periódicos nacionais e internacionais, em um horizonte temporal de sete anos, entre os anos de 2009 e 2015. Para artigos nacionais foram consideradas as bases SPELL ${ }^{\circledR}$, SciELO ${ }^{\circledR}$, a Plataforma de Periódicos da Capes, bem como dois eventos importantes da área, o Congresso da Sociedade Brasileira de Economia, Administração e Sociologia Rural (SOBER) e o Encontro da ANPAD (EnANPAD). Para artigos internacionais, foram eleitos quatro periódicos da área de Administração, Ciências Contábeis e Turismo classificados como A1 pela Capes (Canadian Journal of the Administrative Sciences; Governance (Oxford. Print); Industrial Management + Data Systems; International Journal of Operations \& Production Management) e também três periódicos que publicam sobre o tema classificados como A2 (The 
International Food and Agribusiness Management Review; Journal on Chain and Network Science; British Food Journah. Estes, identificados durante o levantamento bibliográfico.

Dessa forma, o primeiro levantamento dos trabalhos sobre o tema envolveu a seleção daqueles que contivessem no título, palavras-chave ou resumo algum dos termos pré-estabelecidos (Quadro 01). Aqueles que tiveram, foram organizados em forma de planilha no Microsoft Excel $2010{ }^{\circledR}$ e salvos para posterior análise. Nesta primeira etapa, todos os artigos foram tabulados com o intuito de evitar a duplicação dos artigos. Foi analisado, neste momento, um total de 4.485 artigos.

Em seguida, foi realizada uma leitura prévia dos artigos, buscando identificar se eles buscavam discutir sobre a "coordenação" dos sistemas. Ao final, esse levantamento chegou a um total final de 82 artigos. Por fim, foi feita uma segunda leitura buscando identificar as informações referentes aos indicadores préestabelecidos com base em Silva e Bianchi (2001) e Kobashi e Santos (2008). Tais indicadores podem ser observados no Quadro 02.

\section{Quadro 02: Indicadores de análise}

\begin{tabular}{|c|c|c|}
\hline \multicolumn{3}{|l|}{ INDICADORES } \\
\hline Quantitativos & Metodológicos & Teóricos \\
\hline Revista & Abordagem: Qualitativa/Quantitativo & Bases Teóricas \\
\hline Qualis & $\begin{array}{l}\text { Finalidade: Teórico/Empírico } \\
\text { Tipo }\end{array}$ & Citação de Autores \\
\hline & Método & Subtema \\
\hline Palavids-cilave & Tipo de Dados: Secundários/Primários & Resultados \\
\hline Objeto & $\begin{array}{l}\text { Procedimento para Coleta de Dados } \\
\text { Técnica de Análise dos Dados }\end{array}$ & Sistema Convencional/Diferenciado \\
\hline
\end{tabular}

Fonte: Adaptado de Kobashi e Santos (2008) e Silva e Bianchi (2001)

Por fim, a última etapa consistiu na análise qualitativa dos dados por meio de um exame descritivo e analítico combinando perspectivas quantitativas (porcentagens, frequência, contagem etc.) e qualitativas (descrição analítica dos dados matemáticos). Essa análise teve como objetivo obter maior rigor dos resultados e a identificação de pesquisas futuras (CHUEKE; AMATUCCI, 2015).

\section{ANÁLISE DOS DADOS}

Do total de 82 artigos encontrados, 62 são nacionais e 20 internacionais. Apesar dos termos de busca estarem associados à sistemas convencionais e diferenciados, verificou-se que, tanto os artigos 
nacionais quanto os internacionais, estudaram majoritariamente Sistemas Convencionais. Com relação aos estudos sobre Sistemas Diferenciados, verificou-se que $72 \%$ foram nacionais e $28 \%$ internacionais. Observou-se que as pesquisas que focam em Sistemas Diferenciados têm representatividade nos estudos (30\% do total de estudos), indicando que é um campo emergente de análises. Sendo assim, as próximas seções se referem aos estudos não somente de sistemas diferenciados, mas também, convencionais.

\subsection{INDICADORES QUANTITATIVOS DOS ARTIGOS ANALISADOS}

Foram realizadas análises comparativas entre os trabalhos nacionais e internacionais por meio de porcentagem, buscando equipará-los em relação ao número reduzido de meios de comunicação pesquisados para estudos internacionais. Entre os nacionais, mais de $50 \%$ deles foram publicados em eventos, o que mostra que nem todos os artigos apresentados em eventos seguiram efetivamente para futuras publicações. Considerando as revistas brasileiras, aquela com maior número de artigos publicados $(14,52 \%)$ foi a Organizações Rurais \& Agroindustriais, com nove artigos; em segundo lugar a Revista de Administração - RAUSP (6,45\%) e a Revista de Economia e Sociologia Rural $(6,45 \%)$, com quatro artigos; em terceiro lugar a Gestão e Produção (4,84\%), com 3 artigos. Nota-se que duas entre essas quatro revistas trabalham com temas voltados para a área do agronegócio. Outros dez artigos, os quais representam 14,49\% do total, foram publicados nas revistas: Gestão \& Regionalidade, Organizações Rurais \& Sociedade, Revista de Administração Mackenzie (RAM), Revista Eletrônica de Administração (REAd), Revista Brasileira de Administração Científica, Revista Brasileira de Marketing, Revista de Administração da Unimep, Revista de Economia e Administração e Revista Gestão Organizacional.

Dentre os internacionais encontrados, verificou-se que os trabalhos foram majoritariamente divulgados em revistas $A 2$, sendo que aquela com maior número de publicações foi o Journal on Chain and Network Science (45\%), seguido pelo International Food and Agribusiness Management Review (25\%) e pelo British Food Journal (20\%). Os outros 2 artigos encontrados foram publicados nas revistas Industrial Management \& Data Systems e International Journal of Operations \& Production Management. Nota-se que, assim como os nacionais, esses jornais são especializados em publicações voltadas ao agronegócio, e no caso dos internacionais, também redes e cadeia produtivas.

Considerando os artigos nacionais publicados em Revistas Científicas (29), foi possível concluir que eles foram em sua maior parte (65\%) publicados em revistas classificadas como B1. Outras avaliações foram: A2 (14\%), B2 (\&\%), B3 (7\%), B5 (7\%). Quanto aos internacionais, eles foram publicados majoritariamente em revistas $A 2$, indicando que tanto para trabalhos nacionais quanto internacionais, há poucos estudos divulgados em meios considerados com a avaliação máxima. 
Os artigos nacionais foram todos elaborados por autores brasileiros, sendo dessa forma 100\% originados no Brasil. Já com os internacionais, os países com maior número de autores com publicações no tema são: Holanda (26,09\%), Brasil (21,74\%), Estados Unidos (13,04\%) e Alemanha (8,7\%). Os outros países encontrados (7 países) somam 30,45\% do total, o que representa um autor por país. A informação de que o Brasil se encontra em segundo lugar no número de publicações é um dado que complementa a reflexão de que o Brasil é um país com forte participação na produção de conhecimento acerca do tema proposto.

Quanto ao número de publicações nacionais sobre o tema, até o ano de 2013 o número foi em média 11 artigos por ano. No ano de 2012, e novamente a partir de 2014, esse número baixou para uma média de 6 por ano, ou seja, caiu quase pela metade. Isso mostra que o interesse pelo tema pode ter diminuído no horizonte temporal analisado. Outros fatores explicativos para tal achado é a aceitação das revistas e até mesmo a qualidade dos artigos produzidos. Com relação aos artigos internacionais, o número de publicações por ano não variou significativamente, seguindo uma média de 3 por ano. Neste caso, a queda pelo interesse tanto dos autores quanto das revistas pode não ter acontecido, pelo contrário, pode ter aumentado, uma vez que, no ano de 2015, houve um aumento significativo no número de publicações.

A análise dos objetos possibilitou a compreensão de que os trabalhos internacionais sobre o tema, no período em questão, são principalmente aplicados à compreensão de sistemas especificamente agroindustriais. Aproximadamente 55\% dos objetos dos estudos encontrados se referem ao agronegócio. Petróleo e Engenharia constituem 10\% dos demais objetos, e os 35\% restantes são compostos por objetos relacionados a formas híbridas, organizações coletivas e redes. Os artigos nacionais também contam com uma parcela majoritária de objetos voltados ao agronegócio, uma vez que estes compõem em torno de 85\% do total de objetos. Os outros 15\% integram objetos como cadeias, jogos eletrônicos e biocombustíveis.

\subsection{INDICADORES METODOLÓGICOS DOS ARTIGOS ANALISADOS}

Os indicadores metodológicos analisados foram: a abordagem, as finalidades dos estudos, os métodos adotados, as técnicas de coleta de dados, bem como as de análise de dados. No que se refere à abordagem, foi possivel observar que, entre os artigos nacionais, a principal abordagem adotada foi a de pesquisa qualitativa $(82,26 \%)$, seguido pela abordagem do tipo mista $(16,13 \%)$, e, por fim, em apenas um trabalho, a abordagem quantitativa (1,61\%). Já para os internacionais (20 artigos), em primeiro lugar, assim como os nacionais, a pesquisa qualitativa aparece $(60 \%)$, porém, quando se trata de quantitativas, quando comparado aos nacionais, elas aparecem em maior número (25\%). Essas informações podem ser úteis na compreensão dos tipos de pesquisas realizadas em outros países, indicando orientações. 
A finalidade dos estudos, tanto nacionais quanto internacionais, foi majoritariamente realizar pesquisas empíricas, representando 79,03\% e $80 \%$, respectivamente. Além disso, dos artigos nacionais, 19,35\% representam artigos teóricos, enquanto 1,61\% representam artigos teóricos/empíricos. Dos internacionais, dois artigos tiveram finalidade teórica e outros dois tiveram finalidade teórica/empírica, representando $10 \%$ do total. Essas informações refletem o interesse tanto dos autores quanto das revistas que publicam sobre o tema em pesquisas empíricas em detrimento às teóricas.

Com relação ao método utilizado nos artigos, o mais adotado, tanto por artigos nacionais (29 artigos) quanto internacionais (12 artigos), foi o estudo de caso. $72 \%$ das pesquisas nacionais adotaram tal método e cerca de $76 \%$ das internacionais contaram com o mesmo procedimento. Além do estudo de caso, os principais métodos utilizados, delineados pelos autores, em pesquisas nacionais foram: estudo de caso múltiplo $(10,34 \%)$, método indutivo $(10,34 \%)$, proposta conceitual $(10,34 \%)$ e pesquisa rápida $(6,9 \%)$. Nas pesquisas internacionais, por sua vez, os métodos mais utilizados foram: modelo PLS (8\%), modelos matemáticos e estratégicos (8\%) e proposta conceitual (8\%).

Para a coleta dos dados primários, houve a utilização de uma grande variedade de técnicas. Dos estudos nacionais analisados (70 artigos), 34 contaram em grande maioria com a realização de entrevistas, principalmente as semiestruturadas, aplicadas em 24 artigos. Em seguida, a pesquisa documental e a observação direta foram duas técnicas mais utilizadas, presentes em 10 e 7 artigos, respectivamente. Para os internacionais, sendo esses 23 artigos, utilizou-se as entrevistas em 8 artigos e as entrevistas semiestruturadas em 4 artigos. Os questionários também foram utilizados em 4 estudos. Isso mostra que diferentes países seguem um mesmo padrão no que se refere às técnicas de coleta de dados neste tema.

Por fim, no que se refere às técnicas de análise dos dados, analisou-se 68 artigos nacionais e 20 internacionais, sendo possível concluir que cerca de $50 \%$ desses não mencionaram qual o procedimento adotado para tal. Porém, para os nacionais que apontaram, a principal técnica foi a análise de conteúdo $(17,65 \%)$, estando de acordo com a principal abordagem de pesquisa adotada (qualitativa), bem como as técnicas de coleta de dados primários (entrevistas/documental/observação direta). Quanto aos internacionais, foi possivel observar que não houve um procedimento predominante, sendo que 15\% são técnicas qualitativas e 35\% quantitativas.

Por meio da análise dos indicadores metodológicos, é possível inferir que há uma coerência entre os tipos de dados, dos instrumentos de coleta e análise dos dados, dos tipos de pesquisas entre os trabalhos nacionais e internacionais. Porém, é possível concluir que estudos realizados em outros países tem uma preferência maior por artigos de caráter quantitativo quando comparado aos trabalhos realizados no Brasil. Isso pode ser justificado tanto pelo interesse dos autores, quanto das pesquisas e inclinação do tema para a realização de abordagens quantitativas nos diferentes países. 


\subsection{INDICADORES TEÓRICOS DOS ARTIGOS ANALISADOS}

A partir da leitura dos 82 artigos levantados, foi possível codificar os seus objetivos, visando encontrar quais os subtemas de pesquisa. 54\% dos nacionais tiveram a preocupação de compreender quais os mecanismos de coordenação adotados e a eficiência deles. Entre os temas estiveram: mecanismos de coordenação, especialmente em organizações caracterizadas como redes, coordenação por meio de diferentes teorias, falhas de coordenação, inovação mediante o estabelecimento de formas plurais, competitividade de determinada cadeia em função da coordenação e mecanismos de criação e apropriação de valor. Os 20 artigos internacionais levantados tiveram propósitos semelhantes aos nacionais, uma vez que, 55\% do total levantado procurou compreender os mecanismos de coordenação, 30\% a inovação via coordenação e 15\% dos estudos analisaram a competitividade por meio da coordenação.

Para a compreensão do alcance dos objetivos apontados pelos artigos nacionais, diversas correntes teóricas foram adotadas, sendo que a Teoria dos Custos de Transação foi aquela com maior significância. Aproximadamente $52 \%$ dos estudos se basearam na proposta da Teoria dos Custos de Transação, seguido pela Teoria dos Custos de Mensuração (7\%), a Teoria de Redes (4\%), a Teoria Econômica das Convenções $(4 \%)$ e as teorias sobre formas híbridas (3\%). Os dados sobre os artigos internacionais tiveram resultado semelhante, apontando que a teoria mais adotada pelos autores foi a Teoria dos Custos de Transação (20\%), teorias sobre coordenação $(14 \%)$, teorias da inovação $(9 \%)$, teorias sobre formas híbridas (6\%), e Teoria de Redes (6\%). Tais informações são relevantes, pois apontam que existem diferentes teorias que procuram tratar a coordenação em sistemas diferenciados e convencionais, e que diferentes países seguem as mesmas teorias para a compreensão de problemas parecidos. Isso mostra que há coerência daquilo que se busca e que, a partir do consenso nas teorias, os caminhos traçados podem ser aprimorados cada vez mais a partir da consolidação de tais teorias.

Um dado importante obtido por meio da análise das teorias, é que todos os artigos que se basearam na Teoria dos Custos de Mensuração utilizaram também a Teoria dos Custos de Transação, contudo, o inverso não é verdadeiro. Somente $6 \%$ dos estudos que tiveram como base a Teoria dos Custos de Transação utilizaram em complemento a Teoria dos Custos de Mensuração. Isso mostra que pesquisas que as utilizam complementarmente são poucos, o que abre margens para novas discussões que utilizem ambas, pois tal ação permite resultados mais completos e robustos.

Em consenso com os achados sobre as teorias utilizadas nos trabalhos, os autores mais citados estão de acordo com as mesmas. Analisando todas as 1.226 citações encontradas nos referenciais teóricos, os autores mais citados nos artigos nacionais em ordem decrescente foram: Oliver Williamson, Ronald Coase, Décio Zylbersztajn, Claude Ménard, Azevedo, Douglass North, Yoram Barzel e Elizabeth Farina. Todos os autores se referem a pelo menos uma das teorias utilizadas como base teórica, com 
predominância da Economia dos Custos de Transanção. O mesmo acontece com os estudos internacionais: Oliver Williamson, Claude Ménard, Sérgio Lazzarini, Thompson, Hobbs e Young, Uzzi e Bradach, e Eccles e Ronald Coase.

Por fim, mediante uma categorização dos resultados encontrados, foi possível sintetizar quais os principais resultados e quais as principais implicações no que se refere à Coordenação de Sistemas Diferenciados. Os resultados dos trabalhos nacionais apontam que a coordenação é fruto de diversos elementos, como a eficiência dos mecanismos institucionais (CALEMAN; ZYLBERSZTAJN, 2010; QUADROS, 2011; CALEMAN; ZYLBERSZTAJN, 2012; BARBOSA; CÂNDIDO, 2013; CUNHA et al., 2013); a adoção de formas plurais de governança em função das diversas características presentes nas transações (MELLO; PAULILLO, 2010; CARRER et al., 2011); a adoção de contratos formais (MARTINS; SOUZA, 2014); formas híbridas como modo de alcançar a coordenação por meio da comunicação, da troca de conhecimento entre os agentes e do espírito cooperativo (SCHULTZ et al., 2009; CASTRO et al., 2011; DÖRR; GROTE, 2011; JOÃO; LOURENZANI, 2011; LEITE et al., 2010; PEDRO; PROTIL, 2011; ALMEIDA et al., 2013; ALMEIDA; MACHADO FILHO, 2013; FERREIRA et al., 2013; SILVEIRA; OLIVEIRA, 2013; MACHADO et al., 2014; SILVEIRA et al., 2014); a confiança como redutora de comportamento oportunista e criação de reputação (MALAFAIA et al., 2009; SOUZA et al., 2010; SPROESSER et al., 2010; FABBRO et al., 2015); a inovação como condicionante dos mecanismos de coordenação (RANIERI et al., 2011); e a inovação condicionada por mecanismos de coordenação (JARDIM et al., 2013a, JARDIM et al., 2013b).

Outros trabalhos concluem, em acordo com as propostas teóricas, que sistemas diferenciados exigem estruturas de governança mais complexas (DÖRR; GROTE, 2009; SORIO; FAGUNDES, 2009; BOUROULLEC; PAULILLO, 2010; SILVEIRA; OLIVEIRA, 2013; NOGUEIRA et a., 2015; RISSO et al., 2015; SILVA; FOSCACHES, 2015), e que, diante de elevada especificidade de ativos, mecanismos de coordenação mais complexos também se fazem necessários (BRANDÃO et al., 2009; MELLO; PAULILLO, 2009; AVELHAN; SOUZA, 2010; JORIS; VILPOUX, 2013; SOUZA; BÁNKUTI, 2011; FOREST et al., 2015).

As conclusões dos trabalhos internacionais apresentaram alguns resultados semelhantes, porém, foi possivel identificar uma tendência não observada nos nacionais. Os estudos internacionais apontam para a importância da geração de confiança para melhor coordenação (GARBADE et al., 2015); para a certificação como mecanismo de coordenação (BAHLMANN; SPILLER, 2009; VINHOLIS et al., 2014); para a importância da eficiência das instituições no alcance da coordenação (CALEMAN; ZYLBERSZTAJN, 2011); para a adoção de formas plurais na busca de coordenação (CARRER et al., 2014); pela indicação de que estruturas mais complexas fazem-se necessárias diante de elevada especificidade de ativos (MELLO; STRANDHAGEN, 2015; PASSUELLO et al., 2015); e, principalmente, pela adoção de formas híbridas como alternativas em busca da coordenação (SAUVÉE, 2013; BRITO et al., 2015). Nesse ponto, em complementaridade com os resultados nacionais, grande parte dos trabalhos internacionais apontam 
para a importância das organizações em redes na troca de conhecimento, interesses compartilhados, o espírito cooperativo e a confiança entre os agentes na busca de inovação (TEPIC et al., 2011; NIJHOFFSAVVAKI et al., 2012; RAMPERSAD et al., 2012; TEPIC et al., 2012; PIRES et al., 2013; GARBADE et al., 2015). Essas análises apontam que os grupos que se organizam dessa forma têm maior capacidade de realizar inovações, seja pelo compartilhamento de custos, de conhecimento, de escala e escopo, entre outros. 0 quadro 03 sintetiza os principais resultados.

Quadro 03: Principais resultados encontrados

\begin{tabular}{|c|c|}
\hline \multicolumn{2}{|c|}{ Principais resultados nacionais } \\
\hline Eficiência dos mecanismos institucionais & $\begin{array}{l}\text { CALEMAN; ZYLBERSZTAJN, 2010; QUADROS, 2011; CALEMAN; } \\
\text { ZYLBERSZTAJN, 2012; BARBOSA; CÂNDIDO, 2013; CUNHA et al., } 2013\end{array}$ \\
\hline Adoção de formas plurais de governança & MELLO; PAULILLO, 2010; CARRER et al., 2011 \\
\hline Adoção de contratos formais & MARTINS; SOUZA, 2014 \\
\hline $\begin{array}{l}\text { Formas híbridas como modo de alcançar a coordenação } \\
\text { por meio da comunicação, da troca de conhecimento } \\
\text { entre os agentes e do espírito cooperativo }\end{array}$ & $\begin{array}{l}\text { SCHULTZ et al., 2009; CASTRO et al., 2011; DÖRR; GROTE, 2011; JOÃO; } \\
\text { LOURENZANI, 2011; LEITE et al., 2010; PEDRO; PROTIL, 2011; ALMEIDA } \\
\text { et al., 2013; ALMEIDA; MACHADO FILHO, 2013; FERREIRA et al., 2013; } \\
\text { SILVEIRA; OLIVEIRA, 2013; MACHADO et al., 2014; SILVEIRA et al., 2014 }\end{array}$ \\
\hline $\begin{array}{l}\text { Confiança como redutora de comportamento } \\
\text { oportunista e criação de reputação }\end{array}$ & $\begin{array}{l}\text { MALAFAIA et al., 2009; SOUZA et al., 2010; SPROESSER et al., 2010; } \\
\text { FABBRO et al., } 2015\end{array}$ \\
\hline $\begin{array}{l}\text { Inovação condicionada por mecanismos de } \\
\text { coordenação }\end{array}$ & RANIERI et al., 2011; JARDIM et al., 2013a, JARDIM et al., 2013b \\
\hline $\begin{array}{l}\text { Sistemas diferenciados exigem estruturas de } \\
\text { governança mais complexas }\end{array}$ & $\begin{array}{l}\text { DÖRR; GROTE, 2009; SORIO; FAGUNDES, 2009; BOUROULLEC; PAULILLO, } \\
\text { 2010; SILVEIRA; OLIVEIRA, 2013; NOGUEIRA et a., 2015; RISSO et al., 2015; } \\
\text { SILVA; FOSCACHES, } 2015\end{array}$ \\
\hline $\begin{array}{l}\text { Diante de elevada especificidade de ativos, adoção de } \\
\text { mecanismos de coordenação mais complexos }\end{array}$ & $\begin{array}{l}\text { BRANDÃO et al., 2009; MELLO; PAULILLO, 2009; AVELHAN; SOUZA, 2010; } \\
\text { JORIS; VILPOUX, 2013; SOUZA; BÁNKUTI, 2011; FOREST et al., } 2015\end{array}$ \\
\hline \multicolumn{2}{|c|}{ Principais resultados internacionais } \\
\hline $\begin{array}{l}\text { Importância da geração de confiança para melhor } \\
\text { coordenação }\end{array}$ & GARBADE et al., 2015 \\
\hline Certificação como mecanismo de coordenação & BAHLMANN; SPILLER, 2009; VINHOLIS et al., 2014 \\
\hline $\begin{array}{l}\text { Importância da eficiência das instituições no alcance } \\
\text { da coordenação }\end{array}$ & CALEMAN; ZYLBERSZTAJN, 2011 \\
\hline Adoção de formas plurais na busca de coordenação & CARRER et al., 2014 \\
\hline $\begin{array}{l}\text { Indicação de que estruturas mais complexas diante de } \\
\text { elevada especificidade de ativos }\end{array}$ & MELLO; STRANDHAGEN, 2015; PASSUELLO et al., 2015 \\
\hline $\begin{array}{l}\text { Formas híbridas como alternativas em busca da } \\
\text { coordenação. }\end{array}$ & SAUVÉE, 2013; BRITO et al., 2015 \\
\hline
\end{tabular}

Fonte: Dados da pesquisa 
É importante ressaltar que não houve estudos nacionais que buscaram tratar como a organização em redes possibilita a realização de inovações. Dessa forma, essas informações podem ser relevantes, pois representam uma oportunidade emergente de pesquisas, seja pela urgência de investigações na área, pelo elevado crescimento de organizações que se organizem coletivamente, pelo interesse das revistas internacionais ou mesmo dos autores.

\section{CONCLUSÃo}

À guisa de conclusão, é possivel afirmar que o presente trabalho atendeu ao objetivo proposto de realizar um levantamento da produção de conhecimento acerca do tema "Coordenação de Sistemas Agroalimentares" nos últimos sete anos, além de fornecer contribuições para a compreensão contextualizada da produção científica no que diz respeito aos sistemas agroalimentares, de modo a fundamentar a agenda de pesquisas futuras sobre o tema.

Com base no levantamento bibliométrico realizado, foi possível concluir algumas proposições. A partir da comparação das pesquisas nacionais e internacionais, identifica-se que, quando comparado aos sistemas convencionais, os trabalhos sobre sistemas diferenciados ainda têm pouca produção, não havendo baixa ou alta significativa em algum momento. Dessa forma, pode-se inferir que ainda há espaço para discussões em sistemas diferenciados em ambos os contextos, nacionais e internacionais.

As principais abordagens teóricas adotadas em diferentes países são a Teoria dos Custos de Transação, a Teoria dos Custos de Mensuração, a Teoria de Redes e outras teorias que buscam tratar sobre coordenação. Os autores mais citados foram coerentes com as teorias escolhidas, o que indica que os artigos foram coesos nas discussões teóricas. Os trabalhos internacionais têm maior interesse nas correntes teóricas que busquem tratar a coordenação de formas coletivas por meio da cooperação, do estabelecimento da confiança, ou seja, de coordenação horizontal e vertical (LAZZARINI et al., 2001). Isso mostra que, no Brasil, há espaço para pesquisas que tratem do mesmo objetivo, pois são poucas as que analisam sobre esse viés.

Tais informações estão de acordo com as conclusões encontradas acerca dos subtemas e resultados, uma vez que os mesmos apresentaram semelhança em ambas as regiões. Contudo, observou-se uma tendência diferente nos países estrangeiros, pois, diferente do Brasil, os mesmos estão avançados no que se refere a pesquisas em organizações híbridas, como por exemplo, o estudo da adoção de formas híbridas como forma de promover a inovação. Essa informação é útil no que se refere a discussões que contemplem teorias sobre formas híbridas e teorias sobre inovação, no entanto com foco predominante em Teorias de Redes e não mais na Teoria dos Custos de Transação e a Teoria dos Custos de Mensuração. 
No Brasil, somente dois trabalhos contemplaram a integração entre a Teoria dos Custos de Transação e as teorias de inovação, porém, o foco foi diferente, uma vez que estes buscaram tratar de formas plurais como promoção da inovação ao invés de organizações híbridas. Mediante tais dados, é possivel afirmar que não houve estudos que buscaram tratar a Teoria dos Custos de Transação, a Teoria dos Custos de Mensuração, e as teorias de inovação concomitantemente, em quaisquer que fossem os objetos. É possível afirmar, portanto, que existe a possibilidade de desenvolver análises que façam a complementaridade entre as teorias, buscando resultados que possam oferecer contribuições para a coordenação de cadeias produtivas em sistemas diferenciados.

Ambos os horizontes locacionais adotam a abordagem do tipo qualitativa para a realização de investigações nesse tema, o que mostra que para estudos nessa área, as pesquisas qualitativas e suas técnicas de coleta e análise de dados são as mais indicadas. Informação interessante encontrada no presente trabalho é a de que os artigos internacionais, por mais que utilizem pesquisas do tipo qualitativa, adotaram métodos de análise dos dados do tipo quantitativo. Isso pode ser justificado pelo fato de que trabalhos no exterior podem realizar abordagens qualitativas por meio de métodos quantitativos, ou, pelo fato de que muitos artigos não indicaram quais os seus métodos qualitativos, e, por isso, os resultados foram diferentes, sendo esta uma primeira limitação.

Os indicadores quantitativos foram importantes, pois expuseram a aceitação, tanto de meios de comunicação nacionais quanto internacionais, para pesquisas sobre o tema. Informação relevante que merece mais investigações é quanto ao tipo de revista que se interessa por esse tema, sendo que em ambos as regiões analisadas, os estudos sobre o tema foram publicados predominantemente em revistas com classificação do tipo A2 e B1. Apenas dois artigos foram divulgados em revistas do tipo A1. Isso pode estar associado ao interesse das mesmas, bem como dos autores e da qualidade das análises empreendidas.

Como limitações, identificou-se que há uma parte dos artigos que não deixa claro quais os métodos adotados para a condução das pesquisas, bem como as técnicas de coleta e análise dos dados. Outra limitação se refere à forma incorreta com que parte das pesquisas redigiram as obras e autores citados. A escrita incorreta nas citações dos autores ou das obras pode impactar nos resultados do levantamento bibliométrico. Isso porque, para o presente artigo, não foram utilizados softwares para a descrição e análise de dados, os quais auxiliam no tratamento de informações e resultados assertivos. Além disso, em função do espaço da comunicação científica, o artigo não contou com a descrição dos estudos do corpus da pesquisa.

Como pesquisas futuras, sugere-se, além do uso de softwares para a análise dos dados, a ampliação das buscas em bases de dados internacionais como Scopus e Web of Science. Ainda, a realização dessa 
pesquisa de forma fracionada, concentrando em sistemas convencionais e sistemas diferenciados separadamente, pode prover resultados que contribuam para o avanço do campo. Além disso, pesquisas futuras são importantes no que se refere à ampliação das bases de pesquisas internacionais, a fim de aprimorar as análises comparativas entre as investigações nacionais e internacionais.

\section{REFERÊNCIAS}

ALMEIDA, R. P. et al. Desafios para a formação de uma aliança mercadológica na pecuária de corte: um estudo de caso em uma cooperativa no Estado do Paraná. In: LI CONGRESSO DA SOBER, 2013, Belém. Anais... Belém: UFPA, 2013.

ALMEIDA, L. F.; MACHADO FILHO, C. A. P. Sharing competences in strategic alliances: a case study of the Cosan and Shell biofuel venture. Revista de Administração, v. 48, n. 2, p. 359-374, 2013.

AVELHAN, B.; SOUZA, J. P. Estruturas de governança no sistema agroindustrial sucroalcooleiro da região de Araçatuba-SP. In: XLVIII CONGRESSO DA SOBER, 2010, Campo Grande. Anais... Campo Grande, 2010.

BAHLMANN, J.; SPILLER, A. The effect of institutional innovations on food chain governance: a case study on the shifting role of the German QS system from certification to supply chain coordination. Journal on Chain and Network Science, v. 9, n. 2, p. 89-103, 2009.

BÁNKUTI, S. M. S. Eficiência e direitos de propriedade em sistemas agroalimentares diferenciados: um estudo no subsistema de carnes nobres no Paraná. Projeto de pesquisa. Maringá, PR, 2014.

BARBOSA, M. F. N.; CÂNDIDO, G. A. Competitividade em organizações agroindustriais: aplicação metodológica em empresa do setor sucroalcooleiro no estado da Paraíba. Gestão \& Regionalidade, v. 29, n. 86, p. 61-76, 2013.

BARCELLOS, J. O. J. et al. Bovinocultura de corte frente a agriculturização no Sul do Brasil. In: CICLO DE ATUALIZAÇÃO EM MEDICINA VETERINÁRIA, 11., 2004, Lages, SC. Anais... Lages: Centro Agroveterinário de Lages, 2004. p.13-30.

BARZEL, Y. Organizational forms and measurement costs. Journal of Institutional and Theoretical Economics, 161, p. 357-373, 2005.

BOUROULLEC, M. D. M.; PAULILLO, L. F. Governanças híbridas complementares aos contratos no comércio justo citrícola internacional. Gestão \& Produção, v. 17, n. 4, p. 761-773, 2010. 
BRANDÃO, F. S. et al. Aliança horizontal na cadeia de carne bovina: relações entre criadores e terminadores de bezerros nas feiras de comercialização no estado do RS. In: XLVII CONGRESSO DA SOBER, 2009, Porto Alegre. Anais... Porto Alegre, 2009.

BRITO, M. M. et al. Horizontal arrangements and competitiveness of small-scale dairy farmers in Paraná, Brazil. The International Food and Agribusiness Management Review, v. 18, p. 155-172, 2015.

BUAINAIN, A. M. BATALHA, M. O. (Orgs.). Cadeia produtiva da carne bovina. Série Agronegócios. v. 8. Brasilia: IICA: MAPA/SPA, 2007.

CALEMAN, S. M. Q. et al. Custos de mensuração e governança no agronegócio: um estudo de casos múltiplos no Sistema Agroindustrial da carne bovina. Organizações Rurais \& Agroindustriais, v. 10, n. 3, 2008.

CALEMAN, S. M. Q.; ZYLBERSZTAJN, D. Contracts and incentives in quality beef chain: analyzing organizational failures. In: XLVIII CONGRESSO DA SOBER, 2010, Campo Grande. Anais... Campo Grande, 2010.

. Failures in incentive transmission along Brazil's quality beef chain. Journal on Chain and Network Science, v. 11, n. 3, p. 251-262, 2011.

Falta de garantias e falhas de coordenação: evidências do sistema agroindustrial da carne bovi-

na. Revista de Economia e Sociologia Rural, v. 50, n. 2, 2012.

CARRER, M. J. et al. Formas plurais de coordenação das transações na cadeia de carne bovina: um estudo de caso no Brasil. In: XLIX CONGRESSO DA SOBER, 2011, Belo Horizonte. Anais... Belo Horizonte, 2011.

Plural forms of governance in the beef industry: a case study in Brazil. British Food Journal, v. 116, n. 4, p. 643-661, 2014.

CASTRO, L. T. et al. Análise da competitividade do APL de piscicultura no lago de Três Marias. Organizações Rurais \& Agroindustriais, v. 13, n. 3, p. 389-402, 2011.

CENTRO DE ESTUDOS AVANÇADOS EM ECONOMIA APLICADA. PIB do Agronegócio. Disponivel em: <https://www.cepea.esalq.usp.br/br/pib-do-agronegocio-brasileiro.aspx>. Acesso em: 12 jul. 2019.

CHUEKE, G. V.; AMATUCCI, M. O que é bibliometria? Uma introdução ao fórum. InternexT - Revista Eletrônica de Negócios Internacionais da ESPM, v. 10, n. 2, p. 1-5, mai./ago., 2015. 
CUNHA, C. F. et al. Análise da complexidade nas estruturas de governança entre supermercados e produtores agrícolas convencionais e orgânicos no Brasil e nos Estados Unidos: a influência do custo de transação e de mensuração. Revista de Administração, São Paulo, v. 48, n. 2, p. 341-358, jun. 2013.

DÖRR, A. C.; GROTE, T. Understanding the marketing chain of mango and grapes in the São Francisco Valley. In: XLVII CONGRESSO DA SOBER, 2009, Porto Alegre. Anais... Porto Alegre, 2009.

Marketing chain analysis: a case study of the melon sector in Rio Grande do Norte State in Brazil. Organizações Rurais \& Agroindustriais, v. 13, n. 3, p. 377-388, 2011.

FABBRO, J. R. et al. Relacionamento com fornecedores no setor têxtil: um estudo das estruturas de governança entre empresas de confecção e fornecedores. In: XXXIV ENANPAD, 2015, Belo Horizonte. Anais... Belo Horizonte, 2015.

FERREIRA, G.C. BARCELLOS, M. D. Alianças estratégicas em cadeias agroindustriais: estudo de caso na cadeia da carne bovina. XXVIII ENANPAD, 2004, Curitiba. Anais... Curitiba, 2004.

FERREIRA, G. C.; PADULA, A. D. Gerenciamento de cadeia de suprimentos: Novas formas de organização na cadeia da carne bovina do Rio Grande do Sul. Revista Administração Contemporânea, Rio Grande do Sul, v. 6, n. 2, p. 167-184, 2002.

FERREIRA, M. C. M. et al. Vantagens competitivas na produção leiteira: relações horizontais e verticais como foco de análise. In: LI CONGRESSO DA SOBER, 2013, Belém. Anais... Bélem: UFPA, 2013.

FOREST, M. et al. Governança e coordenação na cadeia produtiva da carne bovina: o caso da holding MSX Group. In: LII CONGRESSO DA SOBER, 2015, João Pessoa. Anais... João Pessoa, 2015.

GARBADE, P. J. P. et al. The interplay of structural and relational governance in innovation alliances. Journal on Chain and Network Science, 2015, v. 15, n. 3, p. 1-18.

IPARDES; IBPQ; GEPAI. Análise da competitividade da cadeia agroindustrial da carne bovina no estado do Paraná. Curitiba: IPARDES, 2002, 255p.

JARDIM, G. F. et al. Governance structures and innovation: the case of the brazilian coffee roasting \& griding industry. In: XXXVII ENANPAD, 2013, Rio de Janeiro. Anais... Rio de Janeiro: ANPAD, 2013 a.

. Estruturas de governança interna e a capacidade de inovação em pequenas firmas brasileiras de torrefação e moagem de café. Revista de Administração, v. 48, n. 2, p. 239-253, abr./mai./jun., 2013 b. 
JOÃO, I. S.; LOURENZANI, W. L. Análise SWOT do sistema agroindustrial do amendoim na região de Tupã e Marília - SP. Organizações Rurais \& Agroindustriais, v. 13, n. 2, p. 243-256, 2011.

JORIS, J. L.; VILPOUX, O. F. Transações entre produtores e frigoríficos no setor de ovinos no estado de Mato Grosso do Sul: uma abordagem pela Economia dos Custos de Transação. Organizações Rurais \& Agroindustriais, v. 15, n. 2, p. 220-234, 2013.

KOBASHI, N. Y.; SANTOS, R. N. M. Arqueologia do trabalho imaterial: uma aplicação bibliométricas à análise de dissertações e teses. Encontros Bibli: Revista Eletrônica de Biblioteconomia e Ciência da Informação, Florianópolis, n. esp., $1^{\circ}$ sem. 2008.

LAZZARINI, S. et al. Integrating supply chains and network analysis: the study of netchains. Journal of Chain and Network Science. Wagening Academic Publishers, v. 1, p. 01-22, 2001.

LEITE, A. L. S. et al. Competição no setor de supermercados - uma análise à luz da Economia dos Custos de Transação (ECT). Revista Gestão Organizacional, v. 3, n. 2, art. 8, p. 252-266, 2010.

MACEDO, L. O. B.; MORAES, M. A. F. D. Perfil de governança e a coordenação de alianças estratégicas da carne bovina brasileira. Informações Econômicas, São Paulo, v. 39, n. 3, mar, 2009.

MACHADO, L. H. M. et al. Governança no sistema agroindustrial sucroenergético: estudo de uma usina paulista de gestão familiar. In: XXXVIII ENANPAD, 2014, Rio de Janeiro. Anais... Rio de Janeiro: ANPAD, 2014.

MALAFAIA, C. M. et al. A economia das convenções e os mecanismos de coordenação em ações coletivas: o caso da carne uruguaia certificada. In: XXXIII ENANPAD, 2009a, São Paulo. Anais... São Paulo: ANPAD, 2009.

MARTINS, D. L. C. C.; SOUZA, J. P. Atributos da transação e mensuração e sua influência nas relações entre cooperados e cooperativas em sistemas agroindustriais suinícolas. Revista de Administração Mackenzie, v. 15, n. 3, p. 69-100, 2014.

MELLO, F. O. T.; PAULILLO, L. F. O. Análise do alinhamento entre os atributos das transações e as formas de governanças empregadas na citricultura. Gestão \& Produção, São Carlos, v. 16, n. 4, p. 679-690, dez, 2009.

MELLO, M. H.; STRANDHAGEN, J. O. Analyzing the factors affecting coordination in engineer-to-order supply chain. International Journal of Operations \& Production Management, v. 35, n. 7, p. 1005 $1031,2015$. 
NIJHOFF-SAVVAKI, R. et al. Drivers for innovation in niche pork netchains: a study of United Kingdom, Greece, and Spain. British Food Journal, v. 114, n. 8 p. 1106 - 1127, 2012.

NOGUEIRA, C. R. et al. Sistemas agroalimentares diferenciados: um estudo de coordenação no sistema de leite orgânico no Paraná. In: LII CONGRESSO DA SOBER, 2015, João Pessoa. Anais... João Pessoa, 2015.

OLIVEIRA, T. E. de et al. Alianças mercadológicas e estratégias de diferenciação na cadeia da carne bovina no brasil. Revista Ibero-Americana de Estratégia - RIAE, v. 14, n. 2, abr./jun., 2015.

OLIVEIRA JÚNIOR et al. Estrutura de Governança na cadeia da carne bovina especial: um estudo de caso em uma empresa de Presidente Prudente/SP. Revista Gestão e Desenvolvimento, v. 17, n. 1, jan./abril, 2020.

PASCOAL, L. L. et al. Relações comerciais entre produtor, indústria e varejo e as implicações na diferenciação e precificação de carne e produtos bovinos não-carcaça. Revista Brasileira de Zootecnia, v. 40, p. 82-92, 2011.

PASSUELLO, F. et al. Governance implications of non- GM private standards on poultry meat value chains. British Food Journal, v. 117, n. 10, p. $2564-$ 2581, 2015.

PEDRO, J. J.; PROTIL, R. M. Estratégias de coordenação da cadeia produtiva do trigo em cooperativas: uma análise baseada em diferentes stakeholders institucionais. In: XLIX CONGRESSO DA SOBER, 2011, Belo Horizonte. Anais... Belo Horizonte, 2011.

PIGATTO, G. et al. de. Alianças mercadológicas: a busca da coordenação na cadeia de gado de corte brasileira. In: II WORKSHOP DE GESTÃO DE SISTEMAS AGROALIMENTARES. Anais... Ribeirão Preto: PENSA/ FEA/USP, 1999.

PIRES, A. M. B. et al. The challenge of building effective hybrid organizations in Brazil. Journal on Chain and Network Science, v. 13, n. 1, pp. 83-97, 2013.

PRITCHARD, A. Statistical bibliography or bibliometrics? Journal of Documentation, v. 24, n. 4, p. 348349, 1969.

QUADROS, A. V. C. Estruturas de governança na cadeia produtiva de cafés finos: cooperação horizontal e verticalização para apropriação das quase-rendas. In: XLIX CONGRESSO DA SOBER, 2011, Belo Horizonte. Anais... Belo Horizonte: SOBER, 2011. 
RAMPERSAD, G. et al. IOS adoption in innovation networks: a case study. Industrial Management \& Data Systems, v. 112, n. 9 p. 1366 - 1382, 2012.

RANIERI, C. et al. As inovações tecnológicas na ovinocultura brasileira e seus efeitos na organização do sistema agroindustrial. In: XLIX Congresso da Sober, 2011, Belo Horizonte. Anais... Belo Horizonte, 2011.

RISSO, G. A. et al. Estruturas de governança em sistemas fairtrade no Paraná: implicações sob as perspectivas de custos de transação e custos de mensuração. In: LII CONGRESSO DA SOBER, 2015, João Pessoa. Anais... João Pessoa, 2015.

SAAB, M. S. B. L. M. et al. O desafio da coordenação e seus impactos sobre a competitividade de cadeias e sistemas agroindustriais. Revista Brasileira de Zootecnia, v. 38, p. 412-422, 2009.

SAUVÉE, L. Hybrid governance: sketching discrete alternatives. Journal on Chain and Network Science, v. 13, n. 1, p. 1-9, 2013.

SCHULTZ, G. et al. Agricultura orgânica na região do Vale do Taqueari/RS: análises da estrutura de coordenação e gerenciamento da cadeia produtiva de hortaliças orgânicas. In: XLVII CONGRESSO DA SOBER, 2009, Porto Alegre. Anais... Porto Alegre, 2009.

SILVA, J. A.; BIANCHI, M. L. P. Cientometria: a métrica da ciência. Paidéia, v. 11, n. 20, p. 5-10, 2001.

SILVA, F. Q.; FOSCACHES, C. A. L. Relação entre estratégias mercadológicas e mecanismos de governança: um estudo exploratório na cadeia da bovinocultura de corte. Revista Brasileira de Marketing, v. 14, n. 3, p. 292-303, 2015.

SILVEIRA, J. M. F. J.; OLIVEIRA, A. L. R. O caminho da coexistência: da regulação dos produtos transgênicos à criação dos mercados diferenciados. Organizações Rurais \& Agroindustriais, v. 15, n. 2, p. 253-272, 2013.

SILVEIRA, R. L. F. et al. Formas plurais de governança: uma análise das transações de suprimento entre frigoríficos e pecuaristas. Rev. Econ. Sociol. Rural, Brasília, v. 52, n. 4, p. 761-782, Dec. 2014.

SORIO, A. M.; FAGUNDES, M. B. B. A cadeia agroindustrial da carne ovina em Mato Grosso do Sul. In: XLVII CONGRESSO DA SOBER, 2009, Porto Alegre. Anais... Porto Alegre, 2009.

SOUZA, J. P.; BÁNKUTI, S. M. S. Arranjos contratuais no SAG suinícola no Paraná: uma análise sob a ótica dos custos de transação e de mensuração. In: XLIX CONGRESSO DA SOBER, 2011, Belo Horizonte.

Anais... Belo Horizonte, 2011. 
SOUZA, P. A. R. et al. Estruturas de governança na cadeia produtiva de piscicultura de Dourados-MS: uma abordagem focada na Teoria Econômica das Convenções. Revista de Administração da Unimep, v. 8, n. 2, p. 91-105, 2010.

SPROESSER, R. L. et al. Complementaridades da economia dos custos de transação e da teoria econômica das convenções: um estudo de caso do sistema agroindustrial do mel de Campo Grande - MS. Organizações Rurais \& Agroindustriais, v. 12, n. 2, p. 308-322, 2010.

TEPIC, M. et al. The role of structural and relational governance in creating stable innovation networks: Insights from sustainability-oriented Dutch innovation networks. Journal on Chain and Network Science, v. 11, n. 3, p. 197-211, 2011.

The influence of networking and absorptive capacity on the innovativeness of farmers in the dutch pork sector. International Food and Agribusiness Management Review, v.15, n. 3, p.1-33, 2012.

VINHOLIS, M. M. B. et al. Transaction attributes and adoption of hybrid governance in the Brazilian cattle market. Journal on Chain and Network Science, v. 14, n. 3, p. 189-199, 2014.

ZYLBERSZTAJN, D.; MACHADO FILHO, C. A. P. Competitiveness of meat agri-food chain in Brazil. Supply Chain Management: an international journal, v. 8, n. 2, 2003. 